\title{
Biodegradable nanoparticles of Alginate and chitosan as non-viral DNA oral delivery system
}

\author{
L dia M.D. Gon alves ${ }^{1}$, Ana Cadete $^{1}$,Lara Figueiredo ${ }^{1}$, Cec lia C.R. Calado ${ }^{2}$ and Ant nio J. Almeida ${ }^{1}$ \\ ${ }^{1}$ Faculdade de Farm cia, Universidade de Lisboa iMed.UL \\ Lisboa,Portugal; (lgoncalves@ff.ul.pt / aalmeida@ff.ul.pt) \\ ${ }^{2}$ Faculdade de Engenharia da Universidade Cat lica Portuguesa, \\ Rio de Mouro, Portugal.(PI) \\ (c.calado@fe.lisboa.ucp.pt)
}

\begin{abstract}
The delivery of nucleic acids via the oral route involves overcoming barriers such as degradation of nucleic acids by low pH in the stomach, enzymatic degradation by DNases in the gut, crossing the physical barrier imposed by the mucus layer, cellular uptake, intracellular trafficking and nuclear uptake. As an oral drug carrier system chitosan nanoparticles are ideal, being mucoadhesive, interacting with the anionic sialic acid residues in mucin. In this study, plasmid DNA expressing a humanized secreted Gaussia Luciferase as reporter gene was encapsulated in alginate and chitosan nanoparticles, via a mild ionotropic gelation procedure with sodium tripolyphosphate as a counterion. The nanoparticle system here developed shows effective transfection of different human gastric epithelial cell lines with distinct cell differention. That was confirmed by the expression of luciferase in the different tested conditions, particularly the amount of encapsulated pGLuc.
\end{abstract}

Index Terms plasmid; nanoparticles; chitosan; cell; tranfection

\section{INTRODUCTION}

DNA vaccines provide several important advantages over current vaccine strategies such as live or attenuated viral vaccines or recombinant peptide and protein-based vaccines, because they mimic the effects of natural infection in their ability to endogenously express foreign protein and also due to its unique ability to induce humoral as well as cellular immune responses. In addition, DNA vaccines can be quickly and easily produced with high quality while scale-up of production is much facilitated. Due to their stability when compared to proteins or antigens, there is no need for cold-chain storage [1]. The main limitation is DNA degradation by nucleases. Thus, the development of systems that protect and efficiently deliver DNA has been a major research area for the improvement of DNA based vaccines.

For many pathogens infection occurs through mucosa. Therefore, the production of a specific immune response at mucosal sites is very important for the development of a protection. As an oral drug carrier system, chitosan nanoparticles are ideal, being mucoadhesive, interacting with the anionic sialic acid residues in mucin. Such adhesive properties will enable a better interaction between the carried drug molecule and the membrane epithelium, allowing a more efficient uptake. In addition, it shows a high affinity for negatively charged macromolecules, such as proteins [2] and oligonucleotides [3, 4].

\section{GOALS}

In this work nanoparticles of chitosan and alginate were developed to efficiently incorporate plasmid DNA. The protection under different environmental conditions was evaluated as well as the capacity to protect DNA against nuclease degradation. The capacity to transfect different gastric human cell lines was also studied.

\section{MATERIALS AND MethodS}

\section{A. Materials}

Chitosan of different molecular weight (low molecular weight (CH-LMW, $150 \mathrm{kDa})$; medium molecular weight $(\mathrm{CH}-$ MMW), MW = $450 \mathrm{kDa}$ ) and high molecular weight $600 \mathrm{kDa}$ (CH-HMW), sodium alginate with different viscosities and TPP were obtained from Sigma Aldrich, UK. Plasmid DNA (pDNA) expressing a humanized" secreted Gaussia Luciferase as reporter gene (pCMV-GLuc, 5,7 Kbp (pGLuc), New Englands Biolabs, USA) The plasmid was used to transform E. coli and cell culture expansion was performed on tryptic soy broth (Biokar, France) at $37 \mathrm{C}$ with agitation. Plasmid purification was performed according to the Maxi Quialfilter Kit (Quiagen, Germany). All cell culture reagents were from Invitrogen, UK.

\section{B. Nanoparticle Preparation}

Nanoparticle formation was carried out via ionotropic gelation with sodium tripolyphosphate (TPP) [5]. Briefly, 0.5 $\mathrm{mL}$ of $0.075 \%$ TPP containing different amounts of sodium

\footnotetext{
$1^{\text {st }}$ Portuguese Meeting in Bioengineering, February 2011 
alginate was added to $1 \mathrm{~mL}$ of $0.25 \%$ Chitosan (low molecular weight (CH-LMW, $150 \mathrm{kDa})$; medium molecular weight $(\mathrm{CH}-$ MMW), MW = $450 \mathrm{kDa}$ ) and high molecular weight $600 \mathrm{kDa}$ (CH-HMW) (Sigma Aldrich, Spain) in water under magnetic stirring. The nanoparticles with plasmid (pDNA) were prepared by adding different amounts of pDNA to the solution of TPP containing alginate and this solution was added to the chitosan solution with agitation.

\section{Characterization of Alg/CH nanoparticles}

Particle size and surface charge (zeta potential) of nanoparticles were measured by dynamic light scattering and electrophoretic mobility, respectively (Zetasizer 100HSA, Zetasizer 2000, Malvern Instruments, UK). For particle size analysis, each sample was diluted to the appropriate concentration with filtered purified water. Each analysis was carried out in triplicate at $25 \mathrm{C}$. For the determination of the electrophoretic mobility, samples were diluted with filtered purified water to an appropriate concentration to yield count rate per second (KCps) in the range of 2500-3500. All measurements were performed in triplicate.

The morphological examination of the nanoparticles was carried out by scanning electron microscopy (SEM).

\section{Cell Lines and Plasmid}

Gastric carcinoma cell lines come from the Japanese Physical and Chemical Institute, including MKN28 and AGS (moderately differentiated adenocarcinoma), MNK45 (poorly differentiated adenocarcinoma) and N87 (well differentiated adenocarcinoma). The cells were maintained in Roswell Park Memorial Institute 1640 (MKN28, MKN45 and N87), or Ham s F12 (AGS) medium supplemented with $10 \%$ foetal bovine serum, 100-units $/ \mathrm{ml}$ penicillin, and $100-\mu \mathrm{g} / \mathrm{ml}$ streptomycin in a humidified atmosphere of $5 \% \mathrm{CO} 2$ at $37 \mathrm{C}$. Plasmid DNA expressing a humanized" secreted Gaussia Luciferase as reporter gene (pCMV-GLuc, 5.7 kDa, New England Biolabs, USA) was used. Cell viability was measured by MTT reduction [6]. The expression of luciferase was quantified with a commercial kit (Gaussia Luciferase Assay kit, New England Biolabs, USA).

\section{E. Integrity and Stability}

The physical stability of the formulated complexes was studied using the agarose gel retardation assay. Concentrations of $1 \%$ agarose in TBE buffer were used. Protection of incorporated and adsorbed pDNA against enzyme degradation was studied after incubating the complexes with DNase I. Plasmid integrity following complexation and release from the nanoparticles, and the ability of complexation to protect the plasmid from degradation were also evaluated. Naked plasmid, alginate-chitosan nanoparticle-DNA complexes, and alginatechitosan-adsorbed DNA nanoparticles, prepared at a nanoparticles:pDNA weight ratio of 100:1, 70:1 and 40:1, were treated with 1 UDNase I for $15 \mathrm{~min}$ at $37 \mathrm{C}$, followed by heat inactivation $(60 \mathrm{C} / 15 \mathrm{~min})$ in the presence of $25 \mathrm{mM}$ EDTA. Nanoparticles were then digested through incubation $(4 \mathrm{~h}$, $37 \mathrm{C}$ ) with $20 \mu \mathrm{L}$ chitosanase $(10 \mathrm{U} / \mathrm{mL}$ in $50 \mathrm{mM}$ acetate buffer $\mathrm{pH}$ 5.5). Samples were then run on a $1 \%$ agarose gel containing ethidium bromide.

\section{RESULTS AND DISCUSSION}

\section{A. Nanoparticle Preparation}

Under the mild conditions used alginate was found to be a stabilizing agent to the nanoparticles produced. The size range was 207-398 nm (Fig. 1) and zeta potential varied from +50.1 to $+49.5 \mathrm{mV}$ for nanoparticles produced with low viscosity alginate:low molecular weight Chitosan:TPP (w:w:w) from 0.01:1:0.25 to 1:0.2:0.25. Under physiological $\mathrm{pH}$ conditions nanoparticles present a positive zeta potential which is independent from the alginate proportion, in opposition to the size of the nanoparticles (Fig. 2).

The micrographs shown in Fig. 3 indicate the nanoparticles have a spherical shape and form homogeneous populations. This is consistent with previous observations of similar systems [4, 6, 7]. No difference is observed for nanoparticles with and without incorporated plasmid DNA. Particle size is also consistent with the measurements made by dynamic light scattering.

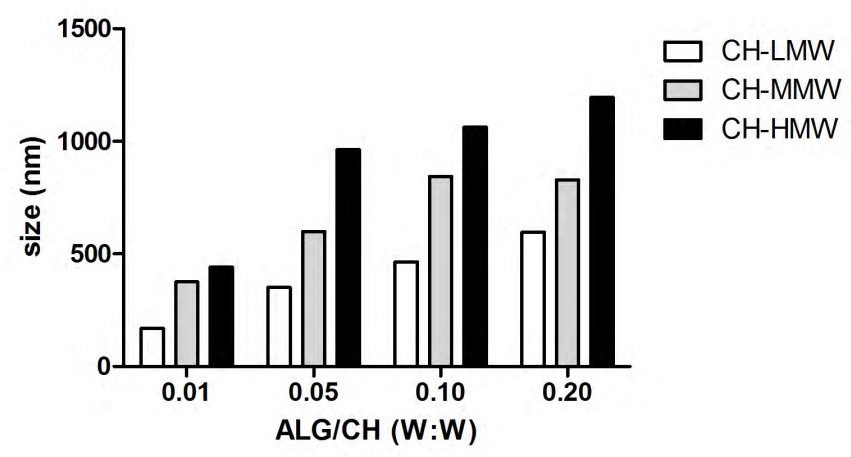

Fig. 1 - Mean Particle size of Alg:CH nanoparticles prepared with different proportions alginate of Low viscosity and chitosan Low Molecular weight_(mean sd; $n=3$ ). 

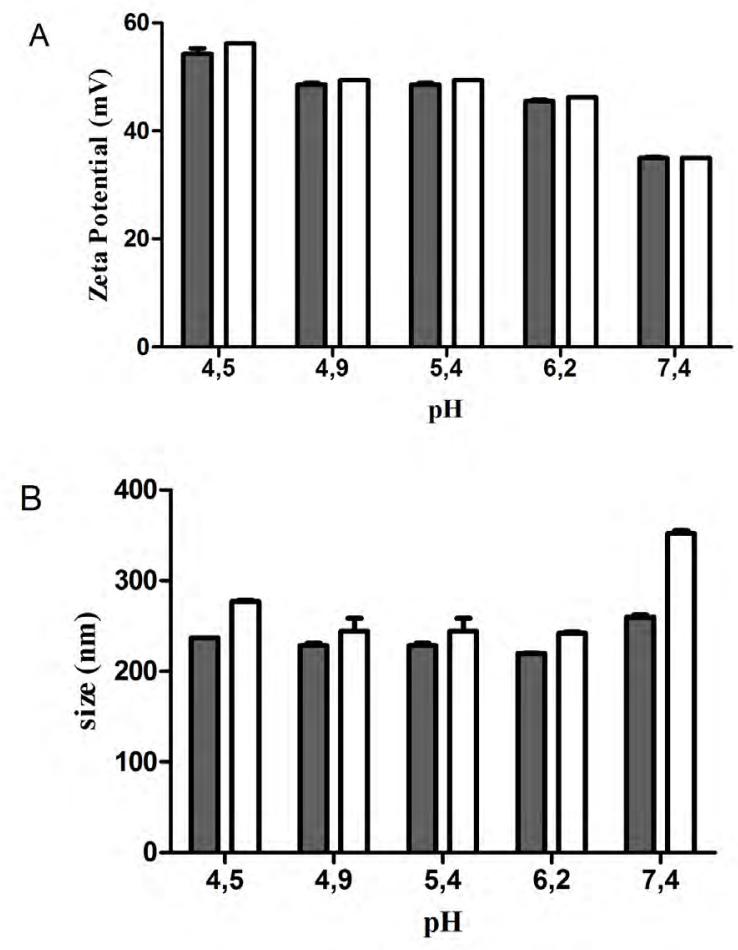

Alg: $\mathrm{CH}(\mathrm{w} / \mathrm{w})$ 0.01:1

Alg: $\mathrm{CH}(w / w)$ 0.05:1

Fig. 2 - Zeta potential (A) and mean particle size (B) of Alg: $\mathrm{CH}$ nanoparticles prepared with different $\mathrm{pH}$ values of the $\mathrm{CH}$ solution $(2.5 \mathrm{mg} / \mathrm{mL})$. Mean $\mathrm{sd} ; \mathrm{n}=3$.
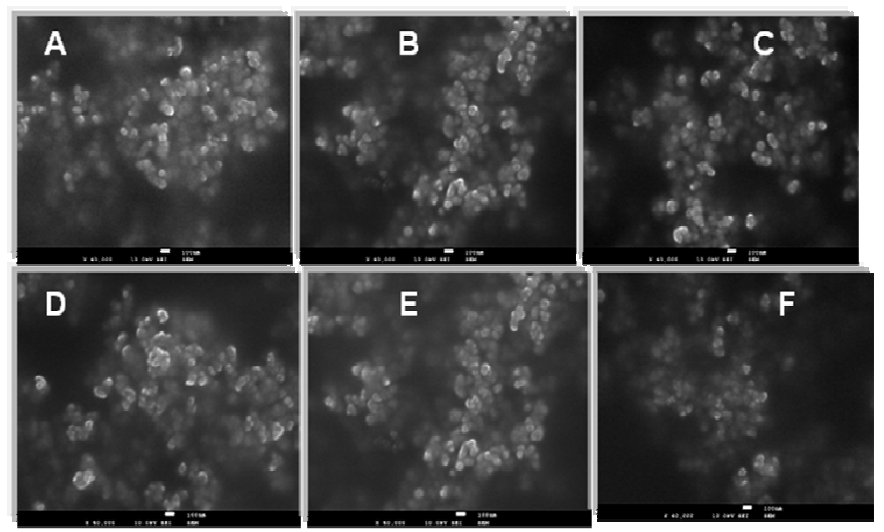

Fig. 3 - Electron scanning micrographs showing alginatechitosan nanoparticles (Alg/CH/ / (w:w) A- 0.01:1; B- 0.02:1; C-0.05:1) and nanoparticles alg/CH 0,05:1 with pDNA D- N/P 5; E- N/P 10;.F- N/P 20.

\section{B. Integrity and Stability}

Chitosan/Alginate nanoparticles can protect the encapsulated plasmid DNA from nuclease degradation (Fig.4) whereas the adsorbed nucleic acid is degraded due to direct exposition to the environment. The nanoencapsulated pGLuc is stable in simulated gastric for one hour and in simulated intestinal medium at $37 \mathrm{C}$ for days (Fig. 5).



Fig. 4 - Agarose gel electrophoresis of alginate-chitosan nanoparticle-DNA complexes to determine protection from DNase and integrity of pDNA. Samples were run on a $0.8 \%$ gel and stained using ethidium bromide: (A) pDNA; (B) adsorbed pDNA to nanoparticles of Alg-CH (0.05:1); (C) Alg-CHpDNA (0.05:1; NP:DNA 100:1); (D) Alg-CH-pDNA (0.05:1;NP:DNA 70:1); (E) Alg-CH-pDNA (0.05; NP:DNA 40:1).

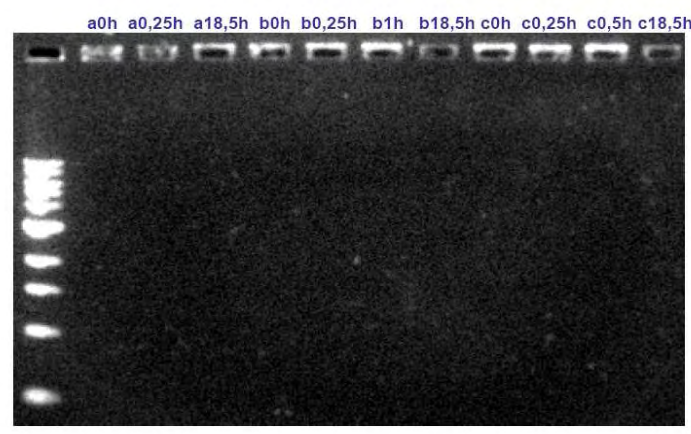

Fig. 5 - Agarose gel electrophoresis of alginate-chitosan nanoparticle-DNA complexes (Alg-CH-pDNA (0.05:1 NP:DNA 70:1) to determine stability at different $\mathrm{pH}$ conditions , at $37 \mathrm{C}$ : (a) $\mathrm{pH} 6.8$, (b) artificial gastric juice without pepsin, pH 1.2; (c) artificial gastric juice with pepsin, pH1.2.

\section{Cell viability and cell transfection}

Nanoparticles containing encapsulated DNA, in different proportions, were able to transfect different cell lines being more effective with AGS cells the less differentiated cell line (Fig. 6). Cell viability measured by MTT reduction assay show that nanoparticles with encapsulated DNA present low toxicity levels, this is in agreement with other method to obtain the same type of nanoparticles [8] and the biocompatibility of the two polymers. 


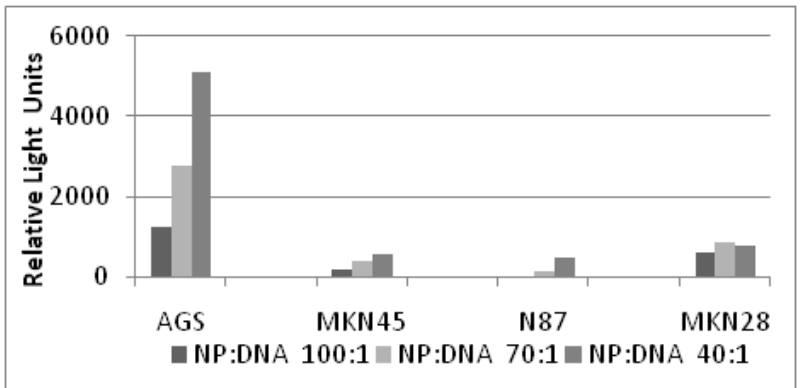

Fig. 6 Luciferase expression by different cells lines transfected with Alg: $\mathrm{CH}$ 0.05:1 containing different proportions of $\mathrm{pDNA}$.

\section{CONCLUSIONS}

Formation of nanoparticles can be achieved under extremely mild conditions. Particle size and surface charge can be modulated by changing the proportion and the specifications of raw materials, namely polymer $\mathrm{MW}$ and $\mathrm{pH}$ of the solutions. The incorporation of pDNA is a more protective method in opposition to the adsorption technique. Alg: $\mathrm{CH}$ nanoparticles are stable under physiological conditions of the intestine and also for short periods of time in the stomach, being a potential oral DNA carrier system. In addition, they present low toxicity and are effective on the transfection of different carcinoma cell lines, showing differences according the surface characteristics of the cells, and thus with the state of differentiation, being more effective for the AGS cell line.

\section{Planned DeVElopments}

This work will be implemented to different plasmids carried out different antigens for immunization studies of mucosal vaccine strategies, namely for Helicobacter pylori vaccines.

\section{ACKNOWLEDGMENTS}

This work was supported by Funda o para a $\mathrm{Ci}$ ncia e Tecnologia (Portugal) (PTDC/BIO/69242/2006).

\section{REFERENCES}

[1] Schalk, J. A., F. R. Mooi, G. A. Berbers, L. A. van Aerts, H. Ovelgonne and T. G. Kimman (2006). "Preclinical and clinical safety studies on DNA vaccines." Hum Vaccin 2(2): 45-53.

[2] Florindo HF, Pandit S, Gon alves LM, Alpar HO, Almeida AJ. (2009). New approach on the development of a mucosal vaccine against strangles: Systemic and mucosal immune responses in a mouse model", Vaccine 27: 1230-1241.

[3] Sato T, Ishii T, Okahata Y. In vitro gene delivery mediated by chitosan. effect of $\mathrm{pH}$, serum, and molecular mass of chitosan on the transfection efficiency. Biomaterials 2001;22(15):2075-80.

[4] Lee M, Nah JW, Kwon Y, Koh JJ, Ko KS, Kim SW. Water-soluble and low molecular weight chitosan-based plasmid DNA delivery. Pharm Res 2001;18(4):427-31.

[5] Calvo, P., Remu n-L pez, C., Vila-Jato, J.L., Alonso, M.J. (1997). Novel hydrophilic chitosan-polyethylene oxide nanoparticles as protein carriers. J Appl Polym Sci. 63:125-32.

[6] Mosmann T. Rapid colorimetric assay for cellular growth and survival: application to proliferation and cytotoxicity assays. J Immunol Methods 1983; 65:55-63.

[7] Yolanda Paraj, I d Angelo, A Welle, M Garcia-Fuentes, and M Jos Alonso. (2010). Hyaluronic acid/Chitosan nanoparticles as delivery vehicles for VEGF and PDGF-BB Drug Delivery, 17(8): 596-604.

[8] de la Fuente, M., Seijo, B., Alonso, M.J. (2008). Novel hyaluron-based nanocarriers for transmucosal delivery of macromolecules.Macromol Biosci. 8:441-50.

[9] Douglas, Kimberly L, Ciriaco a Piccirillo, and Maryam Tabrizian. 2006. Effects of alginate inclusion on the vector properties of chitosan-based nanoparticles." Journal of controlled release : official journal of the Controlled Release Society 115(3): 354-61. 